\title{
The Effect of Sustained Eye Rotation Upon Axial and Peripheral Eye Length in Young, Adult Myopic Subjects
}

\section{Saleh A AlKhaldi ${ }^{1 *}$ and Lyle S Gray ${ }^{2}$}

${ }^{1}$ Department of Ophthalmology, King Saud Medical City, Riyadh, Saudi Arabia

${ }^{2}$ Department of Vision Sciences, Glasgow Caledonian University, Glasgow, UK

*Corresponding Author: Saleh A AlKhaldi, Department of Ophthalmology, King

Saud Medical City, Riyadh, Saudi Arabia.
Received: February 24, 2021

Published: March 10, 2021

(C) All rights are reserved by Saleh A AlKhaldi and Lyle S Gray.

\begin{abstract}
Aim: To determine whether ocular movement can affect the shape of the globe and lead to measurable change in axial and peripheral eye length.

Methods: Ten subjects aged 18 to 30 years old (6M/4F) participated with informed consent. Mean spherical equivalent refractive error was $\leq-1.00 \mathrm{DS}$ with cylindrical refraction <-1.25DC. One drop of tropicamide hydrochloride $1 \%$ was instilled 20 minutes prior to measurement to induce mydriasis and mild cycloplegia. Using IOL Master, eye length was measured centrally and temporally (25 degrees off-axis) in four different positions. Subjects then rotated their eye 25 degrees in the temporal direction to fixate on a target for 10 minutes. After that, the same measurements were repeated.

Results: Prior to rotation, the group mean peripheral eye length was significantly shorter than the central eye length ( $p<0.05$ ). There was no significant variation in central or peripheral eye length due to off-axis fixation, either after the initial eye rotation, or after fixation for 10 minutes at the off-axis point. The difference between central and peripheral eye length was maintained after ten minutes of temporal fixation $(\mathrm{p}<0.05)$.

Conclusions: Peripheral eye length was shorter than central eye length showing the prolate shape associated with myopia. The action of the extraocular muscles on the globe has no significant effect upon the retinal shape assessed by off-axis eye length measurement in myopic subjects.
\end{abstract}

Keywords: Eye Rotation; Eye Shape; Central Eye Length; Peripheral Eye Length; Myopia; IOL- Master

\section{Introduction}

Previous work suggests that peripheral blur signals can influence eye growth, and peripheral blur is the result of a mismatch between the retinal profile and peripheral optical characteristics of the eye [1-6]. Animal models have shown that defocus in local retinal areas can lead to eye growth in that area only [7-12].
Studies have shown that subjects with axial myopia tend to have relative peripheral hyperopia, whereas hyperopic subjects have relative peripheral myopia $[3,4,13]$. The presence of relative peripheral hyperopia is thought to drive ocular growth leading to increased axial length (AL) and myopia [4,5,6,14]. The possible link between myopia development and peripheral hyperopia has directed interest towards investigating peripheral refraction and 
peripheral length (corneo-retinal length along an axis perpendicular to the retinal surface).

A number of studies have been conducted to assess the relation between peripheral refraction and ocular rotation $[2,4,13,15,16]$ by either keeping the eye stationary and rotating the instrument, or by rotating the eye while keeping the instrument stationary. However, there is very limited data available on the relation between peripheral length (PL) and ocular rotation.

MacFadden., et al. (2008) conducted the first study to determine the effect of eye rotation upon the measurement of PL on 23 healthy subjects (13 myopes and 10 hyperopes) using the IOLMaster (Carl Zeiss Meditec, Germany) mounted on a rotating platform. The age of the subjects ranged from 17 to 25 years old and the mean spherical equivalent (MSE) for the myopic group was $-5.08 \mathrm{D} \pm 2.49 \mathrm{D}$, and for the hyperopic group was $+4.77 \mathrm{D} \pm 1.97 \mathrm{D}$. They measured eye length centrally, temporally and nasally across the horizontal meridian up to $40^{\circ}$ under two different experimental paradigms: first, with IOLMaster maintained stationary in a straight position while the subject rotated their eye to fixate off-axis; and secondly, where the subject maintained eye position straight ahead while the IOLMaster was rotated around the eye. Ocular rotation significantly altered the peripheral retinal profile in myopic subjects $(p<0.05)$ but had no effect on hyperopic subjects [17].

Ehsaei., et al. (2013) measured AL and PL up to $30^{\circ}$ temporally and nasally for 27 emmetropic subjects (MSE $=0.10 \pm 0.31 \mathrm{D}$; mean age $=21.67 \pm 3.23$ years) and 52 myopic subjects (MSE $=-5.29 \pm$ $1.86 \mathrm{D}$; mean age $=22.37 \pm 3.77$ years) using a non-contact optical biometer (IOLMaster, Carl Zeiss Meditec, Germany). They found that mean central AL for the emmetropic group was $23.55 \pm 0.48 \mathrm{~mm}$ compared to $23.12 \pm 0.47 \mathrm{~mm}$ (30 degrees temporally) and $23.22 \pm$ $0.49 \mathrm{~mm}$ (30 degrees nasally). For the myopic group, mean central AL was $25.45 \pm 1.10 \mathrm{~mm}$ compared to $24.49 \pm 1.00 \mathrm{~mm}$ (30 degrees temporally) and $24.73 \pm 1.11 \mathrm{~mm}$ (30 degrees nasally), showing that in the myopic group the length of the eye was significantly shorter in the periphery $(\mathrm{p}<0.05)$ [18].

The aim of this study is to determine whether sustained ocular movement generated by the extraocular muscles affects the shape of the eye causing a change in the peripheral eye length in young healthy subjects with axial myopic refractive error.

\section{Methods}

Subjects

Ten healthy myopic adults ( 6 males and 4 females) were recruited from the student population at Glasgow Caledonian University. The age of the participants ranged between 18 and 28 years old (mean $\pm \mathrm{SD}=21.6 \pm 2$.98years). The refractive error ranged between $-2.00 \mathrm{D}$ and $-4.50 \mathrm{D}$ (mean $\pm \mathrm{SD}=-2.92 \pm 0.77 \mathrm{DS}$ ). Subjects were excluded who had any history of ocular or systemic disease, ocular surgery or had astigmatism less than -1.25DC. All participants had best corrected visual acuity of $6 / 6$ or better in both eyes. One drop of tropicamide hydrochloride $1 \%$, was installed in the right eye, prior to measurement, to induce mydriasis. A further drop was installed 20 minutes later if mydriasis was inadequate. The left eye was occluded during the experiment.

The experiment was approved by the School of Health and Life Sciences Ethics Committee and was conducted in accordance with the Declaration of Helsinki for research involving human subjects. All participants completed a consent form and were given information leaflets, after verbal explanation about the nature of the study and any possible consequences. An example of the consent form is given in the appendix section.

\section{Instrumentation}

All measurements were obtained using IOLMaster ocular biometer (Carl Zeiss Meditec, Germany) aligned with the centre of the pupil. This instrument has been found to be accurate and reliable to measure the distance between the cornea and the retina $[19,20]$. To meet the requirements of our experiment, the instrument was specially mounted on a rotating platform with an angular scale to allow it to rotate accurately around the eye.

\section{Fixation targets}

The central fixation target was the internal fixation light of the instrument, whereas the horizontal fixation targets were a number of small LED lights attached to the front of the instrument. The distance between the targets produced eye rotation of $25^{\circ}$ temporally along the horizontal meridian.

\section{Experimental procedure}

Eight measurements were collected from each participant at four different fixation positions (taking 5 readings of eye length 
at each position). At the beginning of the experiment, eye length measurements were taken for each fixation position as baseline measurements. The subjects were then asked to fixate on the temporal target $25^{\circ}$ from the centre for ten minutes.

All the measurements were taken from the right eye, while the left eye was occluded. Furthermore, none of the participants wore any form of optical correction during the procedure. The LED targets are clearly visible and close to the subject, so even if they were not completely clear subjects were instructed to fixate at the centre of the light to ensure steady fixation.

The subject's head was kept stationary all the times by the head and chinrest, and subjects were asked to view each target by turning their eyes only. The time required to complete one set of measurements was approximately 1 minute, with a further minute required for adjusting the position of the eye. The average of 5 readings was calculated for each position at all measurement points (Figure 1 and 2).

Eye length was measured centrally along the visual axis and at $25^{\circ}$ horizontally in the temporal retina. These measurements were repeated with the eye in 4 different positions as follows:

- Baseline measurements were obtained in the central and peripheral locations with the subject fixating upon the central target with no eye rotation.

- The subject then rotated their eye to fixate on the peripheral target placed at $25^{\circ}$ temporally and the measurements were repeated, rotating the IOLMaster as necessary to obtain the measurements.

- The subject was then asked to maintain fixation on the peripheral target with the eye rotated for 10 minutes. Measurements were repeated while the subject continued fixating on the peripheral target.

- Finally, the subject then returned to fixate on the central target and measurements of central and peripheral eye length were repeated at this position.

Figure 1 and 2 demonstrate the infrared beam entering the right eye at $0^{\circ}$ to measure the central AL (A), how both the eye and the instrument were rotated $25^{\circ}$ temporally in order to measure the central AL again (B), eye rotation $25^{\circ}$ temporally with the infrared

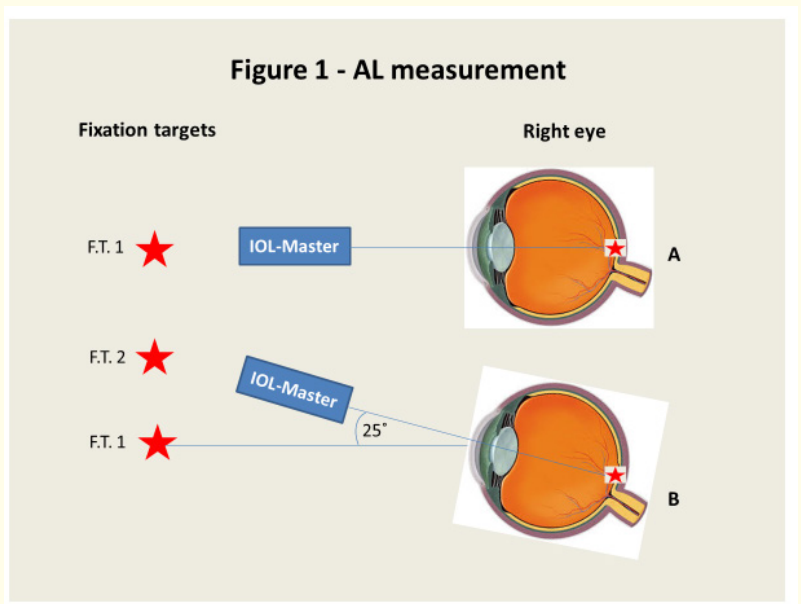

Figure 1: AL measurement.

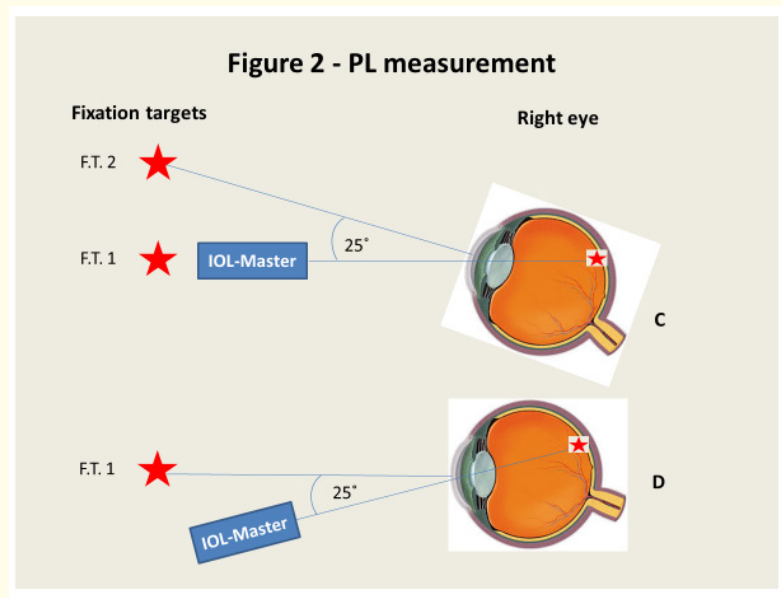

Figure 2: PL measurement.

beam entering the right eye at $0^{\circ}$ to measure the PL (C) and how the instrument was rotated $25^{\circ}$ nasally in order to measure the PL again (D). It can be seen that both beams entered the eye through the central curvature of the cornea and passed close to the nodal point. 


\section{Data analysis}

SPSS software version 22 for Windows (SPSS Inc., Chicago, IL, USA) was used to perform statistical analysis for our data (www. ibm.com/software/analytics/spss/).

A Shapiro-Wilk test was performed to determine the normality which showed the data was normally distributed. A two-way repeated measures ANOVA was used to analyse the data, with time as the repeat measure and retinal location as a factor. Results were considered statistically significant if the $\mathrm{p}$ value is $<0.05$.

\section{Results}

Before subjects were asked to rotate the eye, the central AL varied from $24.10 \mathrm{~mm}$ to $26.20 \mathrm{~mm}$ (mean $\pm \mathrm{SD}=25.06 \pm 0.61 \mathrm{~mm}$ ) and temporal PL varied from $23.49 \mathrm{~mm}$ to $25.43 \mathrm{~mm}$ (mean $\pm \mathrm{SD}=24.31$ $\pm 0.64 \mathrm{~mm}$ ), with all subjects having shorter eye length in the peripheral retina (see table 1 for more details).

\begin{tabular}{|c|c|c|c|c|c|c|}
\hline \multirow{2}{*}{ Position } & \multicolumn{3}{|c|}{ Before temporal fixation } & \multicolumn{3}{c|}{ After temporal fixation } \\
\cline { 2 - 7 } & $\begin{array}{c}\text { No rotation } \\
\text { (mean } \pm \text { SD) }\end{array}$ & $\begin{array}{c}\text { Rotation } \\
(\text { mean } \pm \text { SD) }\end{array}$ & P value & $\begin{array}{c}\text { No rotation } \\
\text { (mean } \pm \text { SD) }\end{array}$ & $\begin{array}{c}\text { Rotation } \\
(\text { mean } \pm \text { SD) }\end{array}$ & P value \\
\hline Central (mm) & $24.91 \pm 0.74$ & $24.92 \pm 0.75$ & $>0.05$ & $24.91 \pm 0.74$ & $24.90 \pm 0.75$ & $>0.05$ \\
\hline Temporal (mm) & $24.22 \pm 0.68$ & $24.19 \pm 0.67$ & $>0.05$ & $24.21 \pm 0.66$ & $24.19 \pm 0.63$ & $>0.05$ \\
\hline AL vs PL & & & $<0.05$ & & & $<0.05$ \\
\hline
\end{tabular}

Table 1: Displays summary of the means for central and peripheral eye length in all positions before and after temporal fixation. Values show mean \pm SD.

A two-way repeated measures ANOVA shows no significant variation in the eye length over time $(F=1.368 ; d f=3,27 ; p=0.274)$. There is a significant difference between group mean central and peripheral eye length at all time points $(\mathrm{F}=38.530 ; \mathrm{df}=1,9 ; \mathrm{p}<$ 0.0001 ) with central eye length being longer than peripheral. There was no significant interaction between the factors of time and retinal location $(F=0.955 ; \mathrm{df}=3,27 ; \mathrm{p}=0.428)$.

At the beginning of the experiment, the baseline central AL was significantly longer than baseline temporal PL ( $p<0.05$; Figure 3$)$.

Figure 3 shows the relationship between central and peripheral eye length with no eye rotation and no temporal fixation at the beginning of the experiment.

As expected, there was significant negative relationship between central MSE and AL, where central AL increased with increasing myopia (Figure 4).

Figure 4 shows the significant relationship between refractive error (MSE) and central AL.

The difference between central AL (group mean=24.91 \pm $0.74 \mathrm{~mm}$ ) and temporal PL (group mean=24.21 $\pm 0.66 \mathrm{~mm}$ ) re-

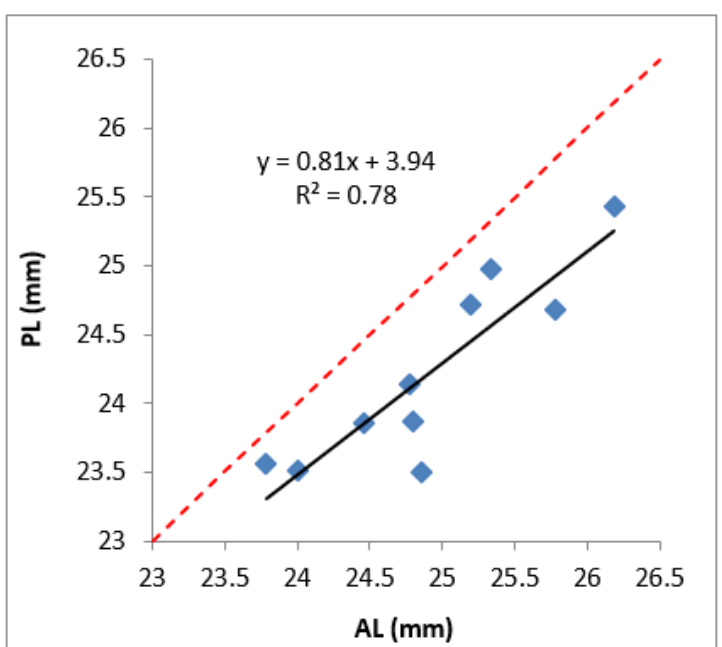

Figure 3

mained significant after 10 minutes of continuous fixation at $25^{\circ}$ temporal to the visual axis ( $\mathrm{p}<0.05$; Figure 5). 


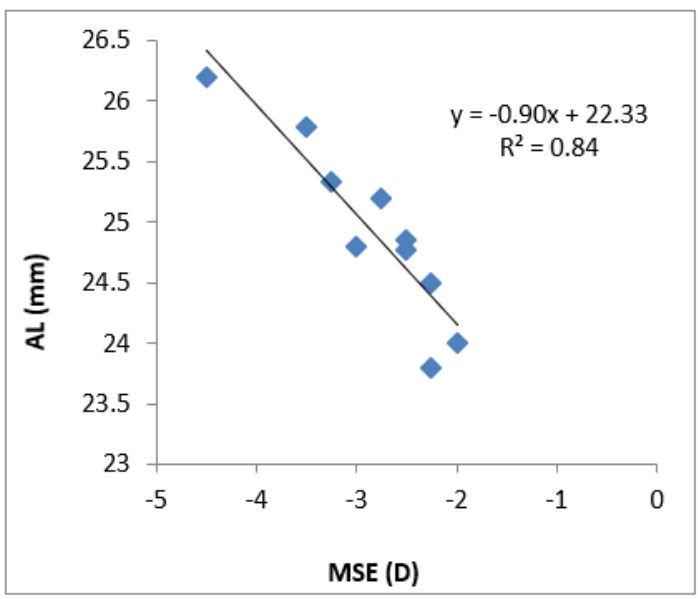

Figure 4

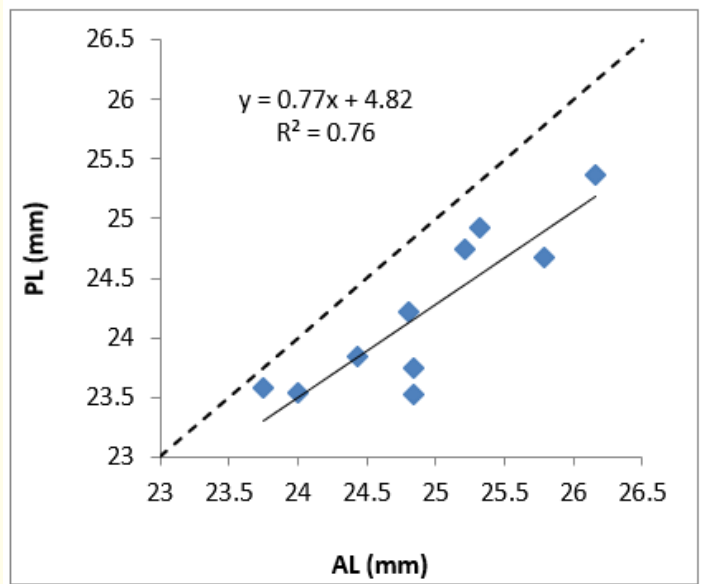

Figure 5

Figure 5 shows the relationship between central and peripheral eye length after temporal fixation with the eye in the straight ahead position.

The effect of eye rotation upon central AL was very small even with 10 minutes of off-axis fixation as can be seen in figure 6.

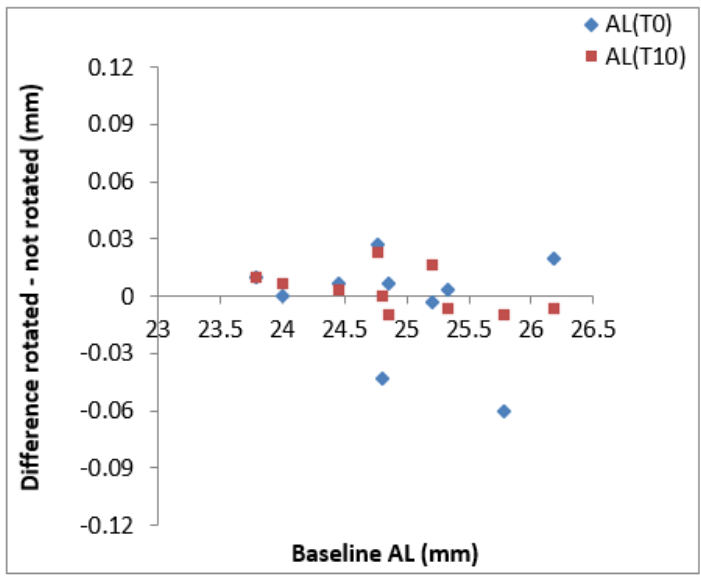

Figure 6

Figure 6 shows the difference between rotated and non-rotated measurement of central AL before and after 10 minutes of off-axis fixation against the baseline measurement of AL.

Eye rotation caused small changes in temporal PL, even after 10 minutes of off-axis fixation, although some intersubject variability is apparent (Figure 7).

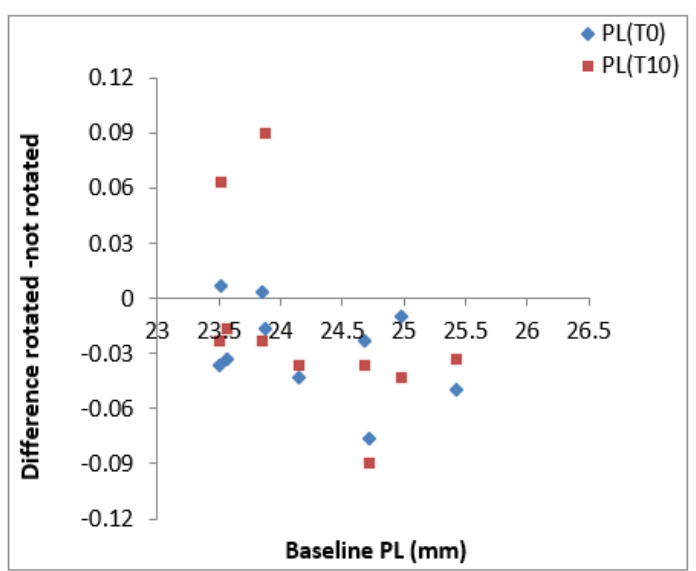

Figure 7 
Figure 7 shows the difference between rotated and non-rotated measurement of PL before and after 10 minutes of off-axis fixation against the baseline measurement of PL.

\section{Discussion}

The results of the current study show little effect of eye rotation upon eye length in either the central or peripheral retinal locations, even after ten minutes of continuous off-axis fixation. The range of myopic subject group in the present study was $-2.00 \mathrm{D}$ to $-4.50 \mathrm{D}$ and this may limit the effect of eye rotation upon retinal profile. This study is one of the first to examine the effect of prolonged eye rotation upon eye length and while there was considerable intersubject variability, some subjects showed substantial changes which suggest that the action upon sclera by the extraocular muscles may be affecting the length of the eye.

Previous studies on the effect of ocular rotation upon peripheral eye length measurement are limited. These studies investigated peripheral retinal profile either by keeping the eye in a fixed position and rotating the instrument, or by keeping the instrument in a fixed position and rotating the eye. The results showed that peripheral retinal profile could be affected by ocular rotation, particularly in subjects with high myopia, such that eye rotation lead to increases in PL and hence an increase relative peripheral myopia for these subjects $[4,17]$.

The aim of the present study was to investigate whether the force generated by the extraocular muscles during eye rotation could affect measurement of eye length in myopic subjects. Examination of the individual changes suggests that certain subjects show increased peripheral length as a result of eye rotation. Previous findings in our lab have shown that ocular rotation could increase PL and that the effect was noticeable with eye rotation $\geq 30$ degrees temporally and in subjects with myopia of more than $-5.00 \mathrm{D}[17]$.

They also found that while ocular rotation altered peripheral retinal shape in myopic subjects, hyperopic subjects showed no significant change. The MSE of the myopic subject group ranged from $-2.00 \mathrm{D}$ to $-9.13 \mathrm{D}$, which is more myopia than in the current study and they also measured further into the periphery (up to $\sim 40^{\circ}$ ). This suggests that repeating the current experiment may be worthwhile in subjects with greater degrees of myopia.
Atchison and Charman (2011) investigated peripheral retinal shape using partial coherence interferometry, with three different schematic eyes (myopic, emmetropic and accommodating). Their results indicated that, apart from the type of schematic eye, the peripheral eye length measurement was not affected up to $30^{\circ}$ of lateral rotation. According to their model, measuring a peripheral point beyond $30^{\circ}$ will cause the measurement beam to be directed away from the nodal point increasing the apparent path length and leading to a value of peripheral eye length which is larger than the true value [21].

There are measurement errors which may affect the measurement of off-axis eye length using IOLMaster due to accommodation changes and variations in the crystalline lens thickness, where the instrument uses the average refractive index of the eye. Using Gullstrand's no. 1 schematic eyes, Atchison and Smith (2004) calculated that $0.4 \mathrm{~mm}$ increase of intra-ocular lens thickness is likely to produce 18 to $26 \mu \mathrm{m}$ for an accommodation of $10.90 \mathrm{D}$ for $\mathrm{AL}$ measurements obtained by the IOLMaster [22]. We restricted our measurements to a point $25^{\circ}$ off-axis, and showed some changes in individual subjects, which again suggest that repeating this experiment in subjects with greater amounts of myopia could be useful.

It has been established that ocular rigidity in myopic eyes is significantly lower than found in emmetropic and hyperopic eyes, and it decreases with increasing myopia [23]. A recent study revealed that the low rigidity of myopic eyes caused a significant decrease in the peak velocity of the eye during saccadic eye movements [24], raising the question of how stable peripheral eye shape is in eyes with greater myopia and lower rigidity.

Previous work has also shown that large amounts of myopia while associated with increased ocular dimensions in all axes $[22,25]$, there is no corresponding increase in orbit size with increasing myopia [26]. It is worth considering therefore whether the movement of a myopic eye is restricted by the limited size of orbital cavity compared to emmetropic eyes or hyperopic eyes.

When evaluating the results of earlier work on peripheral retinal profile to obtain off axis measurements using eye turning, the variations between studies in terms of methods as well as data collection should be considered. As a matter of fact, individual differences in some other factors such as choroidal thickness as well as 
ocular rigidity may also contribute to the variations between previous studies and the current one.

\section{Conclusion}

our data did not show any clear relationship between peripheral eye length and ocular rotation, even with prolonged and continuous off axis fixation. The substantial changes observed in subjects with the largest amount of myopia suggest that the effect of the extraocular muscles upon the sclera during ocular rotation is dependent particularly upon ocular rigidity. We think that the effect of ocular rotation could be more apparent if the experiment was repeated in subjects with higher myopia and possibly with longer duration of eccentric fixation.

\section{Bibliography}

1. Smith EL., et al. "Developmental visual system anomalies and the limits of emmetropization". Ophthalmic and Physiological Optics 19.2 (1999): 90-102.

2. Mutti DO., et al. "Peripheral refraction and ocular shape in children". Investigative Ophthalmology and Visual Science 41.5 (2000): 1022-1030.

3. Mutti DO., et al. "Parental myopia, near work, school achievement, and children's refractive error". Investigative Ophthalmology and Visual Science 43.12 (2002): 3633-3640.

4. Seidemann A., et al. "Peripheral refractive errors in myopic, emmetropic, and hyperopic young subjects". Journal of the Optical Society of America A 19.12 (2002): 2363-2373.

5. Wallman J and Winawer J. "Homeostasis of eye growth and the question of myopia”. Neuron 43.4 (2004): 447-468.

6. Charman WN. "Aberrations and myopia". Ophthalmic and Physiological Optics 25.4 (2005): 285-301.

7. Wallman J., et al. "Extreme myopia produced by modest change in early visual experience". Science 201.4362 (1978): 12491251.

8. Wildsoet CF. "Active emmetropization - Evidence for its existence and ramifications for clinical practice". Ophthalmic and Physiological Optics 17.4 (1997): 279-290.

9. Wallman J and I Adams J. "Developmental aspects of experi- mental myopia in chicks: Susceptibility, recovery and relation to emmetropization". Vision Research 27.7 (1987): 1139-1163.

10. Smith EL., et al. "Peripheral vision can influence eye growth and refractive development in infant monkeys". Investigative Ophthalmology and Visual Science 46.11 (2005): 3965-3972.

11. Smith E L., et al. "Optically Imposed Hyperopic Defocus in the Periphery Can Produce Central Axial Myopia in Infant Monkeys". Investigative Ophthalmology and Visual Science 48.13 (2007): 1533.

12. Smith EL., et al. "Relative peripheral hyperopic defocus alters central refractive development in infant monkeys". Vision Research 49.19 (2009): 2386-2392.

13. Millodot M. "Effect of ametropia on peripheral refraction". American Journal of Optometry and Physiological Optics 58.9 (1981): 691-695.

14. Stone RA and Flitcroft DI. "Ocular Shape and Myopia". In: Annals of the Academy of Medicine Singapore 33.1 (2004): 7-15.

15. Radhakrishnan H and Charman WN. "Refractive changes associated with oblique viewing and reading in myopes and emmetropes". Journal of Vision 7.8 (2007): 5.

16. Radhakrishnan $\mathrm{H}$ and Charman WN. "Peripheral refraction measurement: Does it matter if one turns the eye or the head?". Ophthalmic and Physiological Optics 28.1 (2008): 73-82.

17. Macfadden LA., et al. "Peripheral retinal shape measurements, using the IOLMaster, in myopia and hyperopia". Ophthalmic and Physiological Optics 28.1 (2008).

18. Ehsaei A., et al. "Off-axis partial coherence interferometry in myopes and emmetropes". Ophthalmic and Physiological Optics 33.1 (2013): 26-34.

19. Vogel A., et al. "Reproducibility of optical biometry using partial coherence interferometry: Intraobserver and interobserver reliability". Journal of Cataract and Refractive Surgery 200127.12 (2001): 1961-1968.

20. Connors R., et al. "Accuracy and reproducibility of biometry using partial coherence interferometry". Journal of Cataract and Refractive Surgery 28.2 (2002): 235-238. 
21. Atchison DA and Charman WN. "Can partial coherence interferometry be used to determine retinal shape?" Optometry and Vision Science 88.5 (2011): E601-607.

22. Atchison DA and Smith G. "Possible Errors in Determining Axial Length Changes during Accommodation with the IOL Master". Optometry and Vision Science 81.4 (2004): 283-286.

23. Summers Rada JA., et al. "The sclera and myopia”. Experimental Eye Research 82.2 (2006): 185-200.

24. Alhazmi M., et al. "The effect of ocular rigidity upon the characteristics of saccadic eye movements". Investigative Ophthalmology and Visual Science 55.3 (2014): 1251-1258.

25. Atchison DA., et al. "Shape of the retinal surface in emmetropia and myopia”. Investigative Ophthalmology and Visual Science 46.8 (2005): 2698-707.

26. Chau A., et al. "Is eye size related to orbit size in human subjects?”. Ophthalmic and Physiological Optics 24.1 (2004): 35-40.

\section{Assets from publication with us}

- Prompt Acknowledgement after receiving the article

- Thorough Double blinded peer review

- Rapid Publication

- Issue of Publication Certificate

- High visibility of your Published work

Website: www.actascientific.com/

Submit Article: www.actascientific.com/submission.php

Email us: editor@actascientific.com

Contact us: +919182824667 